\title{
Controlling the transmission at the surface plasmon resonance of nanocomposite films using photonic structures
}

\author{
A. Suarez-Garcia, ${ }^{\text {a) }}$ R. del Coso, R. Serna, J. Solis, and C. N. Afonso \\ Instituto de Óptica, C.S.I.C., Serrano 121, 28006 Madrid, Spain
}

(Received 6 March 2003; accepted 30 June 2003)

\begin{abstract}
The transmission of nanocomposite thin films formed by $\mathrm{Cu}$ nanocrystals embedded in an amorphous aluminum oxide $\left(\mathrm{Al}_{2} \mathrm{O}_{3}\right)$ matrix has been enhanced in the vicinity of the surface plasmon resonance wavelength, by the design of one-dimensional photonic structures. The nanocrystals are distributed in layers, whose separation and periodicity are used to control the optical response of the films. It is found that at the SPR a transmission enhancement up to $36 \%$ can be achieved with respect to a film with an homogeneous distribution of the nanocrystals. These photonic structures have been successfully produced by pulsed laser deposition. (C) 2003 American Institute of Physics. [DOI: 10.1063/1.1605797]
\end{abstract}

Nanocomposite thin films formed by metal nanocrystals (NCs) embedded in a dielectric matrix show attractive optical properties at the surface plasmon resonance (SPR) wavelength due to dielectric and quantum confinement effects. ${ }^{1}$ In the vicinity of this wavelength, the linear optical response exhibits a strong absorption band and the nonlinear optical response shows an enhancement that has been broadly reported. ${ }^{2-4}$ Many applications of these metal nanocrystal composite materials have been proposed taking advantage of the optical response at the SPR, including their use in surface enhanced Raman spectroscopy, ${ }^{5}$ surface enhanced second harmonic generation, ${ }^{2}$ and for the development of sensors ${ }^{6}$ and all-optical switching devices. ${ }^{7}$ The features of the SPR essentially depend on the optical properties of both the metal $\mathrm{NCs}$ and the dielectric host material. The modification of the dimensions and shape of the NCs also induces changes in the intensity and width of the SPR absorption band, which have been extensively studied. ${ }^{1}$ Nevertheless, there are no studies regarding the modification of the optical response around the SPR as a function of the NCs distribution in the matrix.

The aim of this work is to design and synthesize thin films containing metal NCs with a controlled transmission around the SPR wavelength region, using the onedimensional photonic structure concept. A similar idea has been considered to produce metal-dielectric multilayered structures with a spectral range of increased transmittance, as for the case of thin films of $\mathrm{Cu} / \mathrm{SiO}_{2}$ (Ref. 8) and $\mathrm{Ag} / \mathrm{MgF}_{2}$ (Refs. 9 and 10) multilayers. So far, NCs have been only included as "defects" in a dielectric-dielectric multilayer photonic structure to enhance the nonlinear optical response. ${ }^{11,12}$ In this work, it will be shown that the metal NCs can be the building blocks of the photonic structure, and how the transmission around the SPR wavelength can be enhanced by controlling their distribution. This result will have impact on the performance of metal-dielectric nanocomposite devices, such as NCs based nonlinear optical switches, ${ }^{7}$ in which the nonlinear behavior induced by the presence of the NCs has to be preserved. The present ap-

${ }^{a)}$ Electronic mail: asuarez@io.cfmac.csic.es proach will improve the light throughput of the device.

The thin film system selected to model and produce the structures consists of $\mathrm{Cu}$ NCs embedded in an amorphous aluminum oxide $\left(a-\mathrm{Al}_{2} \mathrm{O}_{3}\right)$ matrix, since in previous works this kind of thin films has been successfully produced exhibiting excellent linear and nonlinear optical properties. ${ }^{13,14}$ The thin films were grown on glass substrates by pulsed laser deposition (PLD) technique. An ArF excimer laser was used to alternately ablate high purity $\mathrm{Al}_{2} \mathrm{O}_{3}$ and $\mathrm{Cu}$ targets, in order to grow films with the in depth structure $\mathrm{Al}_{2} \mathrm{O}_{3} / \mathrm{Cu}$ $\mathrm{NCs} / \mathrm{Al}_{2} \mathrm{O}_{3}$. The $\mathrm{Cu} \mathrm{NCs}$ formed in each deposit are distributed on the plane of the film, and their in-depth separation is controlled by modifying the $\mathrm{Al}_{2} \mathrm{O}_{3}$ deposition conditions. Further details on the deposition procedure and structure of the films can be found elsewhere. ${ }^{15,16}$ First, it has been grown a film with the NCs layers homogeneously spaced, whose absorption spectra shows a broad SPR band at 600 $\mathrm{nm} .{ }^{13,14}$ This film will be used as a reference, and will be referred thereafter as sample A. The film is formed by six NCs deposits equally spaced by $a-\mathrm{Al}_{2} \mathrm{O}_{3}$ layers of $7 \mathrm{~nm}$ thickness, as determined from Rutherford backscattering spectrometry (RBS) measurements. This thickness is large enough to ensure that there is no electromagnetic interaction between NCs belonging to different layers. The $\mathrm{Cu}$ content is $9 \times 10^{15}$ at cm $^{-2}$ per layer, corresponding to the development of ellipsoidal NCs with in plane mean diameter of $\sim 5 \mathrm{~nm}$ and average center to center separation of $\sim 12 \mathrm{~nm}$, and a mean height $\leqslant 4 \mathrm{~nm}^{15,16}$

In order to optically model the one-dimensional photonic structures, the thin film is assumed to be formed by alternating $a-\mathrm{Al}_{2} \mathrm{O}_{3}$ and $\mathrm{Cu}$-nanocomposite layers. $\mathrm{A} \mathrm{Cu}-$ nanocomposite layer is defined as the deposited $\mathrm{Cu}$ NCs in each layer plus the $a-\mathrm{Al}_{2} \mathrm{O}_{3}$, which fills in the in-plane space between NCs. The thickness of the nanocomposite layer is assumed to be equal to the mean height of the NCs (4 $\mathrm{nm})$. Since the mean size of the $\mathrm{Cu}$ NCs is much smaller than the wavelength in the visible range, this material can be considered optically as an homogeneous effective medium. The effective refractive index of the defined $\mathrm{Cu}$-nanocomposite layer has been determined by ellipsometry and transmission measurements in sample A. A spectroscopy rotating analyzer 


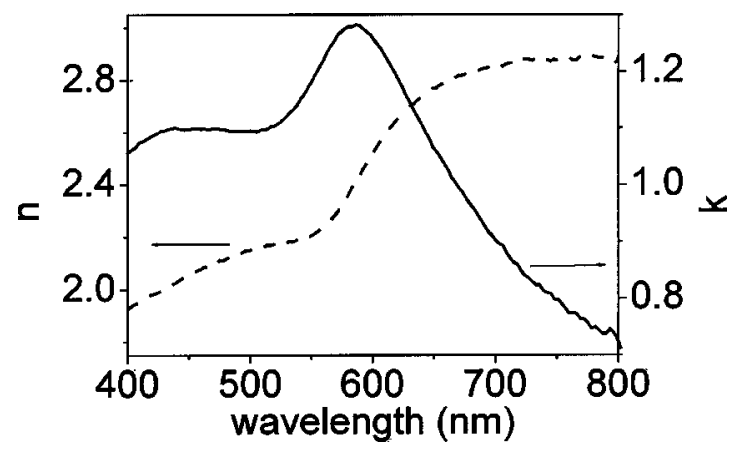

FIG. 1. Effective refractive index, $n(--)$ and $\mathrm{k}(-)$, of the $\mathrm{Cu}-$ nanocomposite layer.

ellipsometer (model VASE, J. A. Woollam Co., Inc.) has been used to measure the ellipsometry parameters $\tan \Psi$ and $\cos \Delta$ in the range $400-800 \mathrm{~nm}$ using steps of $3 \mathrm{~nm}$, for incidence angles $61^{\circ}$ to $75^{\circ}$ at steps of $7^{\circ}$. The transmission has been measured at normal incidence for the same wavelength range. The measured optical response has been modeled as that corresponding to a multilayer structure formed by $a-\mathrm{Al}_{2} \mathrm{O}_{3}$ layers with a thickness of $7 \mathrm{~nm}$, alternated with $\mathrm{Cu}$-nanocomposite layers of $4 \mathrm{~nm}$ for six periods. Since the refractive index of the $a-\mathrm{Al}_{2} \mathrm{O}_{3}$ deposited by PLD has been determined in a previous work, ${ }^{17}$ the complex refractive index of the $\mathrm{Cu}$-nanocomposite layers is the only parameter in the optical model. It has been directly determined using a standard iterative procedure that follows the MarquardtLevenberg algorithm to achieve the best fit of the data simulated for the optical model to the experimental data from both ellipsometry and transmission measurements. ${ }^{18}$ Figure 1 shows the obtained complex effective refractive index of the $\mathrm{Cu}$-nanocomposite layer. The imaginary part of the refractive index shows a band with a maximum at $586 \pm 3 \mathrm{~nm}$, which is close to the SPR broad absorption band reported for films with $\mathrm{Cu}$ NCs embedded in a dielectric matrix. ${ }^{13,19}$ The real part shows the corresponding anomalous dispersion phenomena in the vicinity of the SPR wavelength. ${ }^{1}$

Once the effective refractive index of the $\mathrm{Cu}-$ nanocomposite is known, the one-dimensional photonic structures with an enhanced transmission at $600 \mathrm{~nm}$ have been designed. The inset in the Fig. 2 shows a schematic diagram of the photonic structures. The six $\mathrm{Cu}-$ nanocomposite layers have been organized in two sets of three layers. This set of three layers separated by $7 \mathrm{~nm}$ of $a-\mathrm{Al}_{2} \mathrm{O}_{3}$ is defined as a "photonic unit." It is convenient to use the three layers of nanocomposite instead of one since, in this way, the optical contrast with the $a-\mathrm{Al}_{2} \mathrm{O}_{3}$ is enhanced, i.e., the reflectivity at a single $\mathrm{Cu}-$ nanocomposite $/ \mathrm{Al}_{2} \mathrm{O}_{3}$ interface is only of $11 \%$, whereas a $18 \%$ is obtained when the $\mathrm{Cu}$-nanocomposite layer corresponds to the first layer of the defined photonic unit. The parameters selected to design the photonic structures are then the thickness of the $a-\mathrm{Al}_{2} \mathrm{O}_{3}$ layers that separate the photonic units from the substrate and the film surface $\left(L_{1}\right)$ and between them $\left(L_{2}\right)$. In terms of these parameters, two types of structures, I and II, have been considered in the modeling. Type I has a fixed $L_{2}=7 \mathrm{~nm}$ and a variable $L_{1}$. Structure type I differs only in the $a-\mathrm{Al}_{2} \mathrm{O}_{3}$ thickness $L_{1}$ in respect to the reference sample, which corresponds to the particular case $L_{1}=L_{2}=7 \mathrm{~nm}$. Structure type Downloaded 18 Feb 2010 to 161.111.180.191. Redistribution subject

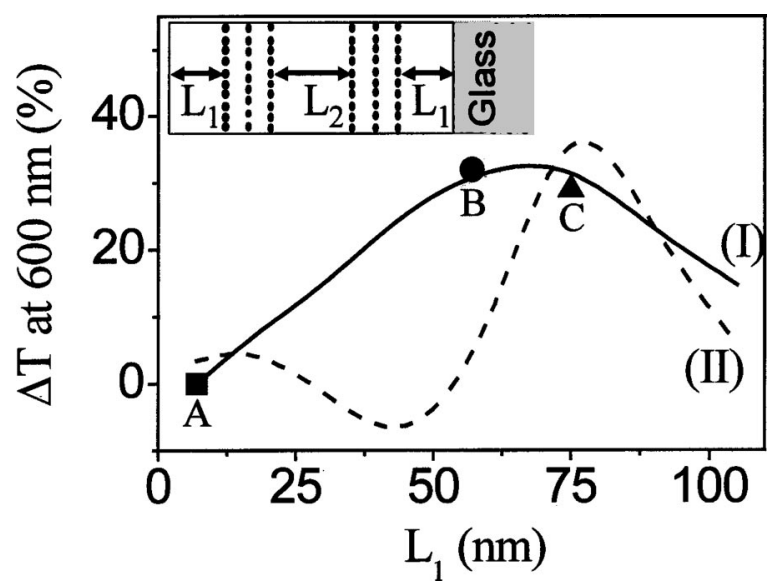

FIG. 2. Calculated relative change $\left[\Delta T=\left(T_{\mathrm{ps}}-T_{r}\right) / T_{r}\right]$ of the photonic structure transmission $\left(T_{\mathrm{ps}}\right)$ respect to the transmission of the reference film $\left(T_{r}\right)$ at $600 \mathrm{~nm}$ as a function of $L_{1}$ for a photonic structure of type I: $L_{2}$ $=7 \mathrm{~nm}(-)$ and type II: $L_{2}=L_{1} / 2(--)$. Experimental values obtained for samples A $(\boldsymbol{\square}), \mathrm{B}(\boldsymbol{\bullet})$, and $\mathrm{C}(\boldsymbol{\Delta})$, are also plotted. Inset schematically shows the structure of the films. Dark dots represent the $\mathrm{Cu}$ NCs and the white background represents the $a-\mathrm{Al}_{2} \mathrm{O}_{3}$. Each block of three layers of $\mathrm{Cu}$ NCs forms a photonic unit. $L_{1}$ and $L_{2}$ are the thicknesses of $a-\mathrm{Al}_{2} \mathrm{O}_{3}$ that are considered as parameters for the design of the photonic structures.

II has a resonator like configuration, described by $L_{1}$ $=L_{2} / 2$. In this way, both structures can be described in terms of a single parameter, $L_{1}$.

Figure 2 shows the relative change in the transmission of structures of types I and II at $600 \mathrm{~nm}$ with respect to that of sample A, calculated as a function of $L_{1}$. The results show that the changes in transmission are very different for the two types of studied structures. Nevertheless, a relative increase above $30 \%$ in the transmission with respect to the reference film is achieved in both cases. For type I the increment is of $32 \%$ for $L_{1}$ equal to $67 \mathrm{~nm}$, whereas for type II the increment is of $36 \%$ for $L_{1}$ equal to $77 \mathrm{~nm}$.

Finally, two thin films, B and C, have been synthesized for the $L_{1}$ values corresponding to the maximum of transmission for types I and II, respectively. They have been grown by PLD, under the same deposition conditions than those used for sample A. They have been subsequently characterized by RBS and ellipsometry, leading both techniques independently to the determination of the $\mathrm{Al}_{2} \mathrm{O}_{3}$ thicknesses $L_{1}$ and $L_{2}$. Table I shows that the results obtained by both techniques are in good agreement and they are also very close to the intended ones.

Figure 3 shows the transmission spectra measured at normal incidence for the three samples [A(reference), B, and C]. Sample A exhibits the typical attributes of a spectrum of $\mathrm{Cu}$ NCs homogeneously distributed in a dielectric medium, showing a decrease of the transmission for a band centered at $600 \mathrm{~nm}$, which corresponds to the SPR. ${ }^{13}$ Samples B and C

TABLE I. Thickness of the $a-\mathrm{Al}_{2} \mathrm{O}_{3}$ layers obtained by ellipsometry $\left(L_{1}, L_{2}\right)$ and RBS $\left(L_{1}^{\prime}, L_{2}^{\prime}\right)$ for the three synthesized samples. Data are given in $\mathrm{nm}$.

\begin{tabular}{ccccc}
\hline \hline Sample & $L_{1}$ & $L_{2}$ & $L_{1}^{\prime}$ & $L_{2}^{\prime}$ \\
\hline A & $7 \pm 1$ & $7 \pm 1$ & $7 \pm 1$ & $7 \pm 1$ \\
B & $57 \pm 6$ & $7 \pm 1$ & $66 \pm 4$ & $7 \pm 1$ \\
C & $75 \pm 8$ & $150 \pm 15$ & $79 \pm 4$ & $158 \pm 8$ \\
\hline \hline
\end{tabular}




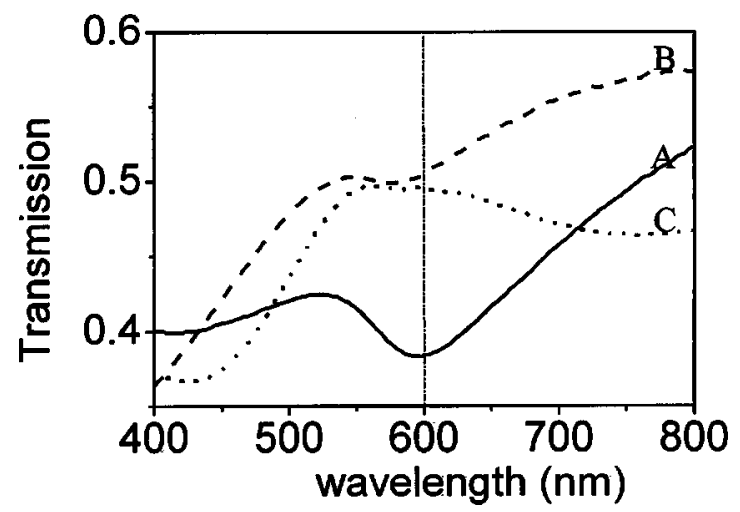

FIG. 3. Transmission spectra of the synthesized samples A (-), B (- - -), C (. . .), as a function of wavelength. A continuous vertical line marks the SPR wavelength.

exhibit a considerable enhancement of the transmission not only at the SPR wavelength, but also in a wide range around it. Sample B shows a reminiscence of the SPR absorption band, while the transmission of sample $\mathrm{C}$ has almost no dependence on the wavelength in this region. These results evidence that it is possible to compensate the typical low transmission at the SPR band by distributing the metal NCs in a one-dimensional photonic structure.

The experimental values of the relative increase in transmission for samples B and C with respect to sample A at 600 $\mathrm{nm}$ have been included in Fig. 2. The increments are 32\% and $29 \%$ for samples B and C, respectively. The slight difference observed with respect to the model calculations is in part due to the small variation that exits in between the thicknesses obtained in the grown films, $L_{1}$ and $L_{2}$, (see Table I) and the intended values. In addition, the ellipsometry measurements have shown that the effective refractive index of the $\mathrm{Cu}$-nanocomposite layers for each sample shows relative variations within $13 \%$ from sample A to C. In spite of these slight differences, the experimental transmission enhancements are very close to the optimum values that can be achieved according to the model calculations.

In conclusion, one-dimensional photonic structures containing $\mathrm{Cu}$ NCs have been modeled. First, the layers containing the metal NCs have been optically characterized using an effective refractive index. Subsequently, by means of an appropriate selection of the distribution of the $\mathrm{Cu}-$ nanocomposite layers, photonic structures have been designed and optimized to achieve an enhancement in the transmission at the SPR wavelength up to $36 \%$, respect to the film in which the $\mathrm{Cu}$ NCs are homogeneously distributed. The photonic structures have been finally produced by PLD, exhibiting an optical response in good agreement with that predicted from the models.

D. E. Hole is acknowledged for performing RBS measurements. This work has been supported by Project No. TIC2002-03235, CICYT (Spain).

${ }^{1}$ U. Kreibig and M. Vollmer, Optical Properties of Metal Clusters (Springer, Berlin, 1995).

${ }^{2}$ R. Antoine, M. Pellarin, B. Palpant, M. Broyer, B. Prével, P. Galleto, P. F. Brevet, and H. H. Girault, J. Appl. Phys. 84, 4532 (1998).

${ }^{3}$ K. Uchida, S. Kaneko, S. Omi, C. Hata, H. Tanji, Y. Asahara, A. J. Ikushima, T. Tokizaki, and A. Nakamura, J. Opt. Soc. Am. B 11, 1236 (1994).

${ }^{4}$ D. Ricard, P. Roussignol, and C. Flytzanis, Opt. Lett. 10, 511 (1985).

${ }^{5}$ S. Nie and S. R. Emory, Science 275, 1102 (1997).

${ }^{6}$ A. J. Haes and R. P. Van Duyne, J. Am. Chem. Soc. 124, 10596 (2002).

${ }^{7}$ G. I. Stegeman and E. M. Wright, Opt. Quantum Electron. 22, 95 (1990).

${ }^{8}$ R. S. Bennink, Y. Yoon, R. W. Boyd, and J. E. Sipe, Opt. Lett. 24, 1416 (1999).

${ }^{9}$ M. Bloemer and M. Scalora, Appl. Phys. Lett. 72, 1676 (1998).

${ }^{10}$ M. Scalora, M. J. Bloemer, A. S. Pethel, J. P. Dowling, C. M. Bowden, and A. S. Manka, J. Appl. Phys. 83, 2377 (1998).

${ }^{11}$ N. Tsurumachi, M. Abe, M. Arakawa, T. Yoda, T. Hattori, J. Qi, Y. Masumoto, and H. Nakatsuka, Jpn. J. Appl. Phys., Part 2 38, L1400 (1999).

${ }^{12}$ N. Tsurumachi, S. Yamashita, N. Muroi, T. Fuji, T. Hattori, and H. Nakatsuka, Jpn. J. Appl. Phys., Part 1 38, 6302 (1999).

${ }^{13}$ J. M. Ballesteros, R. Serna, J. Solis, C. N. Afonso, A. K. Petford-Long, D. H. Osborne, and R. F. Haglund, Jr., Appl. Phys. Lett. 71, 2445 (1997).

${ }^{14}$ R. de Nalda, R. del Coso, J. Requejo-Isidro, J. Olivares, A. Suarez-Garcia, J. Solis, and C. N. Afonso, J. Opt. Soc. Am. B 19, 289 (2002).

${ }^{15}$ R. Serna, C. N. Afonso, C. Ricolleau, Y. Wang, Y. Zheng, M. Gandais, and I. Vickridge, Appl. Phys. A: Mater. Sci. Process. 71, 583 (2000).

${ }^{16}$ R. Serna, J. Gonzalo, A. Suarez-Garcia, C. N. Afonso, J. P. Barnes, and A. K. Petford-Long, J. Microsc. 201, 250 (2000).

${ }^{17}$ R. Serna, J. C. G. de Sande, J. M. Ballesteros, and C. N. Afonso, J. Appl. Phys. 84, 4509 (1998).

${ }^{18}$ C. M. Herzinger, P. G. Snyder, B. Johs, and J. A. Woollam, J. Appl. Phys. 77, 1715 (1995).

${ }^{19}$ R. H. Magruder, L. Yang, R. F. Haglund, Jr., J. E. Wittig, and R. A. Zuhr, J. Appl. Phys. 76, 708 (1994). 